Original Research Paper

\title{
Synthesis of Graphene on Conducting Substrate by Electrochemical Deposition Method
}

\author{
Mazin Ahmed Ali Moghazi, Sondos Al Shareef, H.Y. Wong and Mukter Zaman \\ Faculty of Engineering (FOE), Multimedia University (MMU), 63100 Cyberjaya Selangor, Malaysia
}

\section{Article history}

Received: 10-09-2016

Revised: 24-01-2017

Accepted: 20-02-2017

Corresponding Author:

Mukter Zaman

Faculty of Engineering (FOE),

Multimedia University (MMU),

63100 Cyberjaya Selangor,

Malaysaia

Email: mukter@mmu.edu.my

\begin{abstract}
Investigation to optimize the synthesis process of graphene on conducting substrate is essential for many applications. In this investigation suitable approach to extract graphene thin film on a conductive ITO substrate is optimized. This optimization is carried out by electrochemical Reduction of Graphene Oxide (RGO) solution utilizing a repetitive cyclic potential against reference electrode. To do this, Silver chloride reference electrode is employed within a three electrode system at a potential range of (0 to -1.5$) \mathrm{V}$. A number of four consecutive cycles is used to obtain a negative reduction peak. Yet more importantly the material's electrochemical properties were studied by Cyclic Voltammetry (CV) operation. From $\mathrm{CV}$, it is clear that, the surface area with electrodeposition from ITO to $\mathrm{RGO} / \mathrm{ITO}$ and $\mathrm{RGO} / \mathrm{RGO} / \mathrm{ITO}$ rates in a potential window of $(2 \mathrm{~V})$ for (-1 to 1$) \mathrm{V}$ is improved. Moreover, Electrochemical Impedance Spectroscopy (EIS) showed reduced electrode surface internal resistance of the modified electrode that dramatic decreased in comparison from $96.33 \mathrm{k} \Omega$ of $\mathrm{RGO} / \mathrm{ITO}$ to $32 \Omega$ of $\mathrm{RGO} / \mathrm{RGO} / \mathrm{ITO}$. In addition, Charge/Discharge Cycle (CDC) is obtained that showed relatively symmetrical charging property along with its corresponding discharge equivalents viewing rapid charging/discharging process that favors redox process for increased mobility and thus conductivity improvement. Moreover the modified electrode was studied for its physical characteristics using Scanning Electron Microscopy (SEM) that showed uniform reduced graphene sheets deposition with particles diameter ranging from $1.65 \mu \mathrm{m}$ to $635 \mathrm{~nm}$. Atomic Force Microscopy (AFM) was used in contact mode for a square area of $5 \mu \mathrm{m}$ showing increased surface roughness from ITO of $2.773 \mathrm{~nm}$ in comparison to Reduced Graphene Oxide/RGO/ITO $34.93 \mathrm{~nm}$. Results also proved that deposition of multiple layers of Graphene could strongly improve energy storage applications if implemented by allowing the material to hold greater charge in smaller area. This will lead to enhance the power densities far beyond existing electrochemical capacitors in a cost effective eco-friendly manner.
\end{abstract}

Keywords: Reduced Graphene Oxide, Conductive Substrate, Cyclic Voltammetry, Reduction, Electrochemical Impedance, Modified Electrode

\section{Introduction}

Graphene synthesis has been reported in the past years with a diversity of methodological approaches that made it the most desired material in the fields of science and engineering that was for its exhibited extraordinary properties such of which includes its Crystal structure is a $2 \mathrm{D}$ honeycomb lattice of a flat single atom layer with carbon atoms covalently bonded in $\mathrm{sp}^{2}$ hybridization. That can improve energy storage devices and conservation efficiency by its high tensile strength $\sim 1100 \mathrm{GPa}$, Extremely high electronic mobility $200,000 \mathrm{~cm}^{2} /$ V.s and most importantly high electrical conductivity $\sim$ Zero band-gap along with a large surface area $\sim 2630 \mathrm{~m}^{2} / \mathrm{g}$ as compared to other materials (Shao and Wang, 2010), which makes it crucial as the surface area is proportional to the amount of charges that can be stored in a device. Nevertheless this work is time consuming, but it originates to scan rate chosen, which improves the quality of the deposited 
sheets and it can directly be implemented in efficient batteries and supercapacitors applications. Where in the charging rate is rapid in comparison to the discharging rate that is being looked for in new technologies and for energy conversion application. At the same time there has been a great driven growth in the field Electrochemical Energy Storage (EES) systems that Lead to an increasingly demand for efficient energy storage device with minimum carbon footprint energy source that causes less environmental pollution effects and can still be environmentally friendly (Hibino, 2017). As there is a clear gap between storage devices energy and power density that can be bridged with fabricated graphene based materials (Zang and Li, 2011; Chong et al., 2015). From another prospective supercapacitors exists and have attracted a lot of attention lately, as it serves as power sources that have many appealing properties such as rapid charging/discharging rate, long lifetime and energy density of several orders of magnitude higher than that of conventional dielectric capacitors, yet there was a tradeoff between either high energy density or high power density. This problem was proposed to be overcome by implementing graphene in the supercapacitors electrode material (Lia and Weia, 2013; Wang et al., 2013).

This Work sequence starts with the introduction on the research study and literature review, followed by the research methodology and the procedure taken for extracting graphene and electrochemically depositing it on a conductive substrate, followed by electrochemical characterization including (CV, EIS, CDC) techniques along with structural, morphological and elemental composition characterization with (AFM, SEM, EDS) of the modified reduced graphene electrodes.

\section{Materials and Apparatus}

ITO coated glass substrate with $10 \mathrm{ohm} / \mathrm{sq}$ resistivity, 1.0M phosphate buffer $\left(\mathrm{K}_{2} \mathrm{HPO}_{4}\right.$ and $\left.\mathrm{KH}_{2} \mathrm{PO}_{4}\right)$ of $\mathrm{pH} 7.2$, potassium chloride (KCL) and $3 \mathrm{~mm}$ GCE tip and Graphene Oxide Gel of $10 \mathrm{mg} \mathrm{mL}$, in addition to Apparatus used of Metrohm's compact potentiostat/galvanostat (PGSTAT204) with NOVA software by metrohm Malaysia was earlier installed on laptop computer, ultra-sonication (power sonic 405, HWASHIN technology co) were utilized in this experiment to electrochemically.

\section{Methodology}

About $3 \mathrm{~mL}$ of $\mathrm{GO}$ gel was prepared earlier was allowed to dissolve in $45 \mathrm{~mL}$ DI water and were ultrasonicated for $2 \mathrm{~h}$ till obtaining a well dispersed solution of $\mathrm{GO}\left(1.5 \mathrm{mg} \mathrm{mL} \mathrm{mL}^{-1}\right.$ ), followed by adding the 0.1 phosphate buffer solution to the prepared this GO, proceeded by ultra-sonication of the mixture again for
30 min to obtain a homogenous solution that was poured in an $100 \mathrm{~mL}$ NOVA AUTOLAB beaker for the electro-deposition following process as shown in Figure 1 (flow chart) (Wang et al., 2013). Meanwhile, ITO glass substrate samples were being prepared for electrolysis by cleaning carefully to be polished with fine micro cloth and washed in DI water and ethanol sonication for $10 \mathrm{~min}$ and was left to dry for $20 \mathrm{~min}$ at $65^{\circ} \mathrm{C}$. That were finally impurity free ITO glass substrate was attached as the conductive substrate to the working electrode (RED), the Platinum wire as the counter electrode (BLACK) and $\mathrm{Ag} / \mathrm{AgCl}$ saturated reference electrode (Blue) immersed in the prepared mixture of $0.1 \mathrm{M}$ potassium buffer solution $(\mathrm{pH} 7.2$ ) and the obtained $32 \mathrm{~mL}$ of GO $1.5 \mathrm{mg} \mathrm{mL}^{-1}$ as previewed in Figure 2.

Figure 2 shows an overall view of the whole experiment, initially started with (a) Graphene oxide solution, (b) ultrasonicated GO solution and with the implementation of (c) 3-electrode system that were connected to a Potentiostat auto-lab with the electrodes accordingly to the color code respectively.

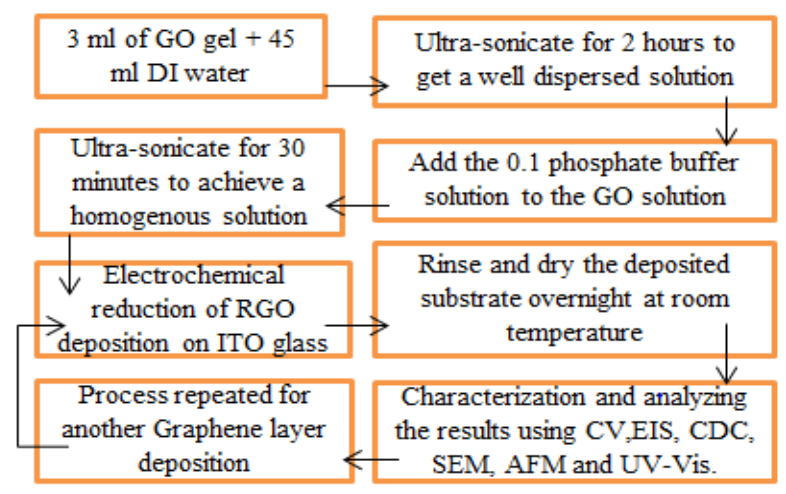

Figure 1. Presents a flow chart of the conducted steps followed in the experiment

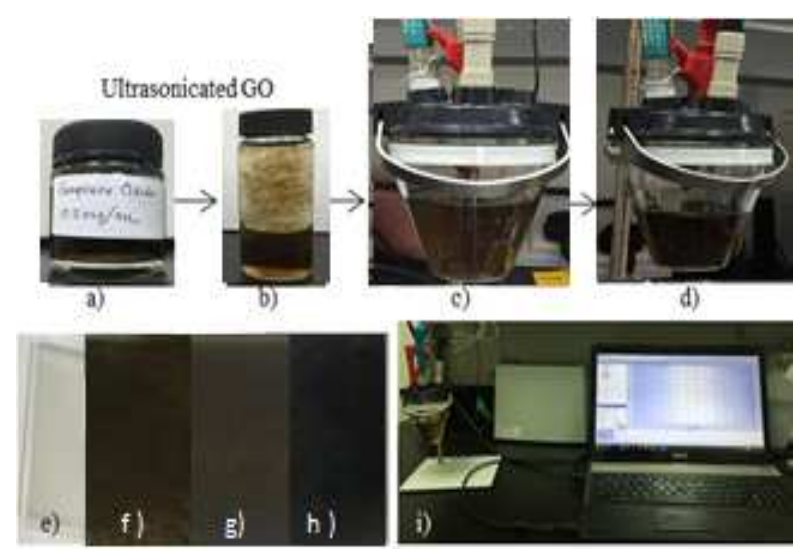

Figure 2. Overall view of the whole experiment connected to NOVA software for the GO reduction process 


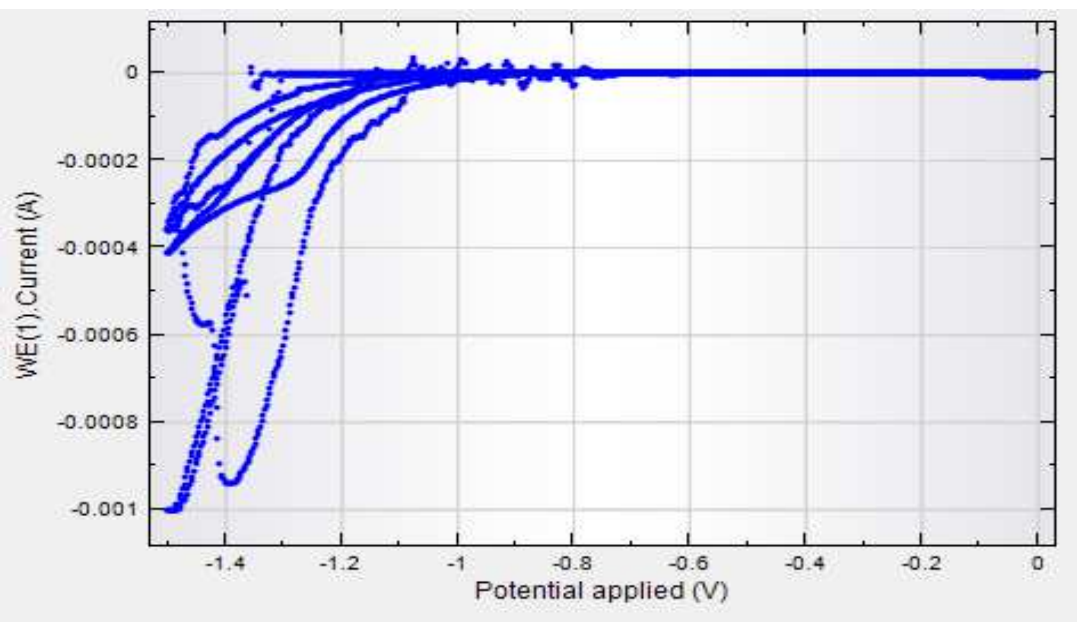

Figure 3. Illustrates 4 cyclic voltammetry cycles of electrochemical deposition of RGO on ITO glass substrate at $1 \mathrm{mV} \mathrm{s}^{-1}$ scan rate

Followed by applying a predefined power supply with $(0.0$ to $-1.5 \mathrm{~V})$ potential range for 4 cycles at $1 \mathrm{mV} \mathrm{s}^{-1}$ which leads to negative reduction peak process, (d) resulting in a reduced $\mathrm{GO}$ that is darker in color for coating of Reduced Graphene Oxide on anode side of the (e) ITO substrate through a reduction process on the oxidized GO, (f) obtaining a layer deposition of RGO on ITO, (g) 2 layers depositions coating of graphene and (h) few layers of RGO on the electrode of ITO working electrode side ( $\mathrm{Yu}$ et al., 2013). In connection made between the PC and metrohm Auto lab potentiostat as a power supply.

Figure 3 illustrates the highest cathodic peak in the first half cycle at almost $-1.44 \mathrm{~V}$, three peaks can be observed in the negative scan of the first cycle.

It is also seen that the first and the second peaks were from the redox pair of oxygen-containing groups on the graphene oxide basal plane that had high stability and was to be reduced while the third peak corresponded to the irreversible electrochemical reduction process (Wang et al., 2013).

\section{Results and Discussion}

\section{UV-Vis Spectroscopy of Graphene Oxide}

Analysis for the samples was carried out from a wavelength ranging from (190-800nm) of aqueous homogenous dispersion of GO collide different samples that were employed for electrochemical deposition process succession that was carried out using GO of 0.5 $\mathrm{mg} \mathrm{mL}^{-1}$ for sample (S1 and S2) and $1.5 \mathrm{mg} \mathrm{mL}^{-1}$ for samples (S3 and S4) along with $0.1 \mathrm{M}$ phosphate buffer solution and a $0.2 \mathrm{M}$ phosphate buffer solution. Four samples were done with different coated number of layers of graphene according to the numbers of cycles applied as shown in Figure 4. Less concentrated samples had a larger amount of inter-lamellar water that is present in stacked GO films and a Hydrogen bond network is formed between the water molecules and the oxygenated groups on GO (Han et al., 2014). Whereas sample 3 was coated with 1 layer and sample 4 with 2 cycles in a concentrated buffer solution with $1.5 \mathrm{mg}$ $\mathrm{mL}^{-1} \mathrm{GO}$ as shown in Figure 4 of thin film deposition.

In common it was found that the light brownish color GO solution color changes in the process of reduction to a light black RGO solution. Which is also a result of elimination of oxygen groups in GO that makes a hydrophobic reduced graphene after the reduction process which greatly reduces the electrochemically accessible surface area of the modified electrode, yet it also increases the capacitive current and stabilization of the electrode. Moreover the reduction process can also be monitored through wavelength red-shifting trends illustrated by UV-Vis analysis which resembles a completed reduction process proven with the peak and hump of S1 that has been redshifted to around $525 \mathrm{~nm}$, while the absorbance peak of $\mathrm{S} 2$ around $350 \mathrm{~nm}$ that was due to $\pi-\pi^{*}$ transitions, originating from C-C bonds (Chong et al., 2015; Lekakou et al., 2012).

\section{Electrochemical CV Characterization of Synthesized Samples}

With a prepared $0.1 \mathrm{M} / 1 \mathrm{KCL}$ electrolyte buffer solution that was chosen due to its satisfactory performance as compared to other electrolytes. Each sample was studied separately under different scan rates influence in a potential window of $(2 \mathrm{~V})$ set between a lower potential of $-1 \mathrm{~V}$ and a higher potential of $1 \mathrm{~V}$ as shown in Figure 5 for (a) RGO/ITO, (b) RGO/RGO/ITO initially starting with a slow scan rate of $10 \mathrm{mV} \mathrm{s}^{-1}$ that was gradually increased to $150 \mathrm{mV} \mathrm{s}^{-1}$. High scan rates cause higher noise levels and less accurate results due to the fact that fast scan rates causes graphene sheets 
agglomeration which is unwanted as it reduces the obtained surface area. Moreover, the anodic peaks shift positively while the cathodic peaks shift negatively for the electrode's internal resistance (Lia and Weia, 2013). The shape of curves is not affected much at a scan rate less than $20 \mathrm{mV} \mathrm{s}^{-1}$, indicating the good stability rate of the electrode material. The results show linear response to current peaks rise that was due to redox confined reactions at the modified electrode's surface (Beitollahi et al., 2014; Han et al., 2014).
Figure 6 makes a comparison of the resultant integrated surface area as more graphene layers are deposited that shows a clear increase in the surface area from ITO to RGO/ITO and RGO/RGO/ITO that also favors charge/discharge along with redox process of electron transfer of increased mobility and thus conductivity improvement on the modified electrode's surface at a fixed slow scan rate in order to eliminate most of the noise present in the background (Devadas et al., 2012).

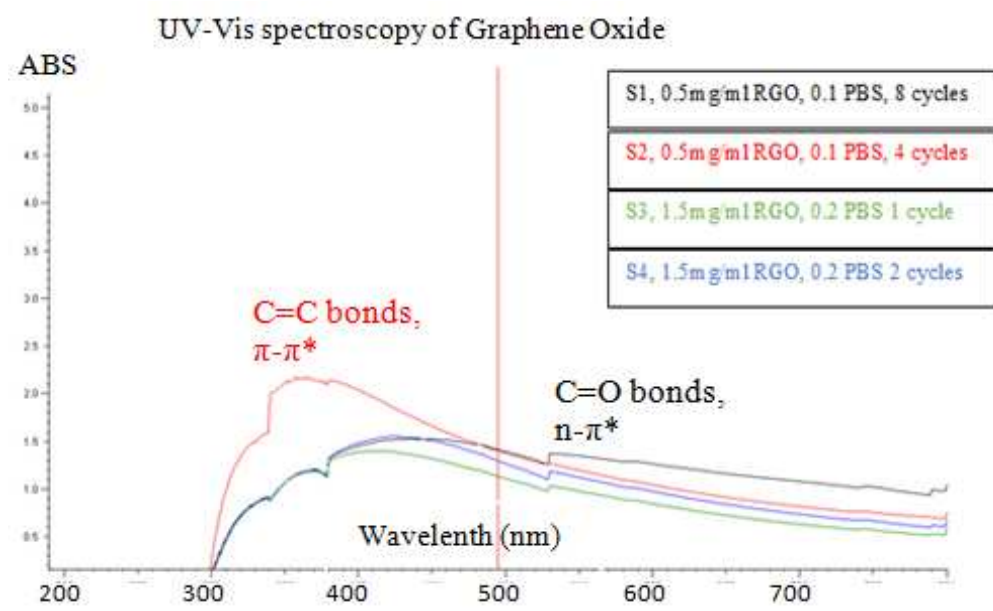

Figure 4. Illustrates the plots of thin film electrochemical deposition of RGO on an ITO glass substrate
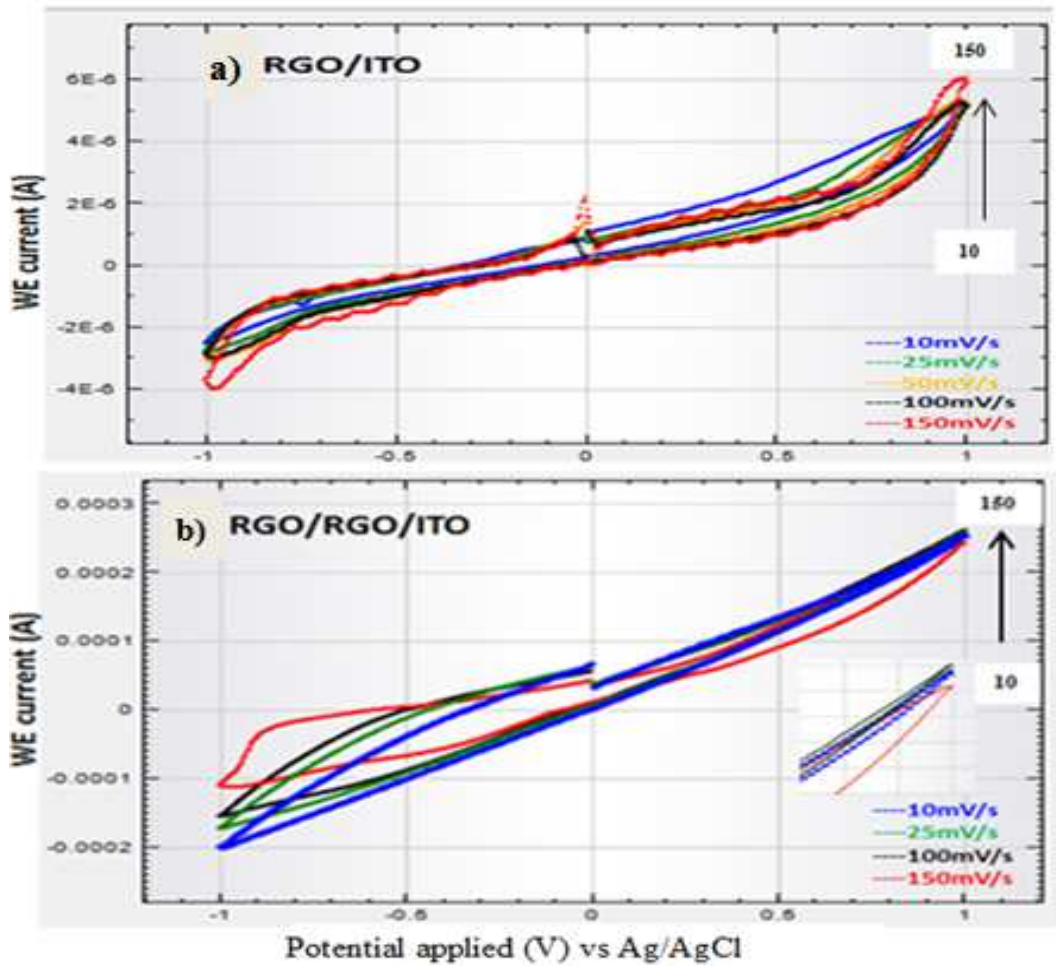

Figure 5. CV plots of different scan rates on differently modified samples (a) RGO/ITO and (b) RGO/RGO/ITO 


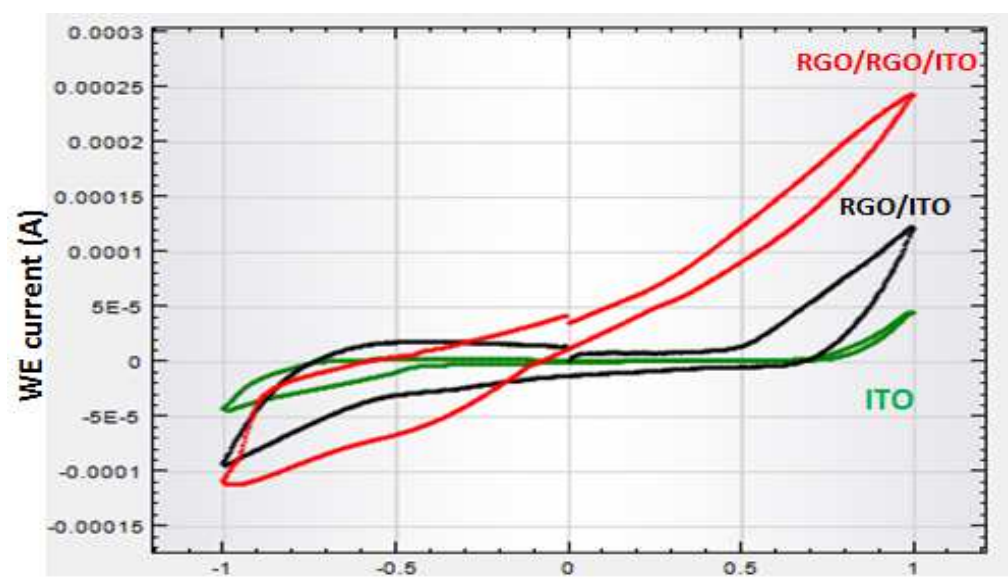

Figure 6. CV curves of ITO, RGO/ITO and RGO/RGO/ITO at a scan rate of $10 \mathrm{mV} \mathrm{s}^{-1}$

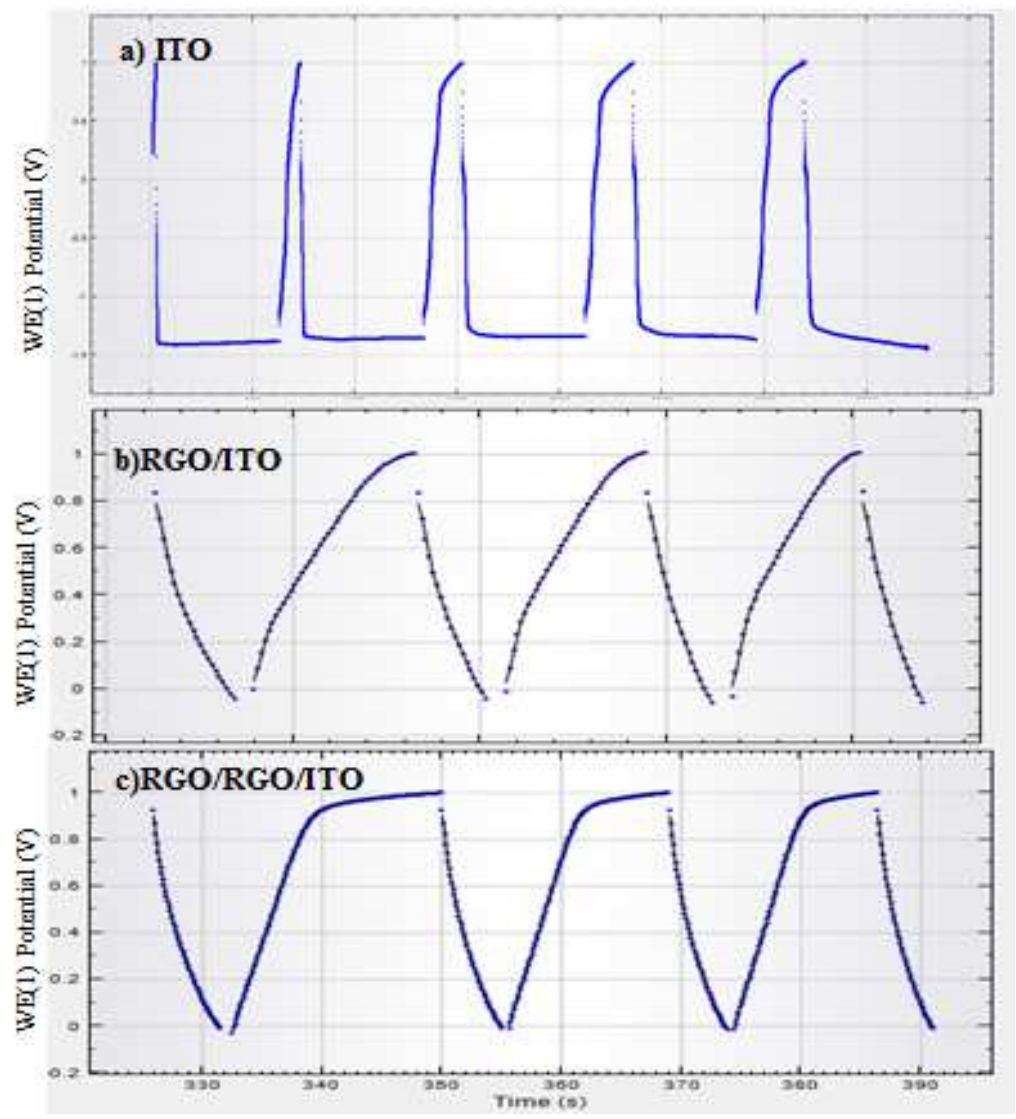

Figure 7. CDC in 0.1M KCL buffer, (a) cycles of bare ITO, (b) RGO on ITO and (c) of RGO/RGO/ITO

\section{Charging Discharging Cycles of Modified Electrodes}

CDC is another important method for testing the electrode's performance capacitance in terms of charging/discharging stability and to compare performance of modified electrode with bare ITO (Beitollahi et al., 2014). The obtained linear voltage to time profiles was recorded in a voltage range of $1 \mathrm{~V}$ potential window for a constant current density that proves enhanced capacitive performance of the modified electrodes. The CDC curves obtain showed relatively symmetrical charging property along with its corresponding discharge equivalents. For comparison of performance between ITO, RGO/ITO and RGO/RGO/ITO verified 5 cycles in an aqueous $0.1 \mathrm{M} \mathrm{KCL}$ buffer solution as shown 
in Figure 7 of CDC linear applied potential in working electrode against time of operation.

The results clearly showed rapid charging/discharging time that conform higher capacitance modified electrodes in addition to conformation of enhancement of electrode position of RGO layers in comparison to bare GCE or ITO that is also justified by good shaped triangle response ( $\mathrm{Li}$ and Östling, 2013; An et al., 2010; Zhang et al., 2012).

\section{Electrochemical-Impedance-Spectroscopy Modified Electrode Internal Systems} of

Analysis corresponding to electron transfer on the studied electrode. Thus EIS characterization was utilized in buffer solution of $0.1 \mathrm{M}$ KCL. An establishment can be made from the results that faster ion transportation was achieved in $\mathrm{RGO} / \mathrm{RGO} / \mathrm{ITO}$ over RGO/ITO electrode, where an internal resistance improvement is also expected for the RGO modified electrode through Nyquist plot that shows the nearer the plot is towards the imaginary axis it is interpreted as the semicircle ratio higher and it defines its ideal capacitive behavior of an electrode in a pre-set frequency range of 0.1 to $100 \mathrm{kHz}$. The semicircle retrieved with NOVA software electrochemical circle fit command option, bode plot and bode where each parameter is well defined as; Rs = Uncompensated resistance value, $\mathrm{Rp}=$ Charge transfer resistance, $\mathrm{CPE}=$ value of the argument of the constant phase element and $\mathrm{N}=$ Exponential value to the constant phase element.

Taking into consideration the Rs value of the circuits from Figure 8-10 for $\mathrm{RGO} / \mathrm{ITO}$ in comparison to $\mathrm{RGO} / \mathrm{RGO} / \mathrm{ITO}$ resembles the charge transfer resistance which showed a dramatic decreased in comparison from 96.33 to $32 \Omega$. This is evidence that RGO layers improve electrode transfer of the electrode with great decline in the internal resistance as well as verified a lower resistance for $\mathrm{RGO} / \mathrm{RGO} / \mathrm{ITO}$ as an electrode of super capacitors device also at low frequencies in comparison to high frequencies.
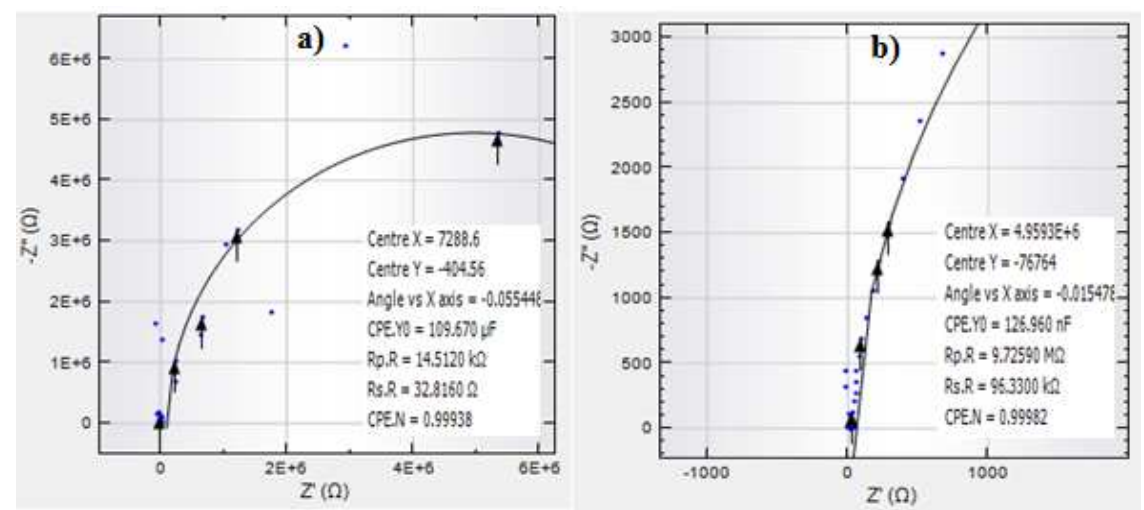

Figure 8. Nyquist semicircle along with equivalent parameters value of circle fit for (a) RGO/ITO and (b) RGO/RGO/ITO

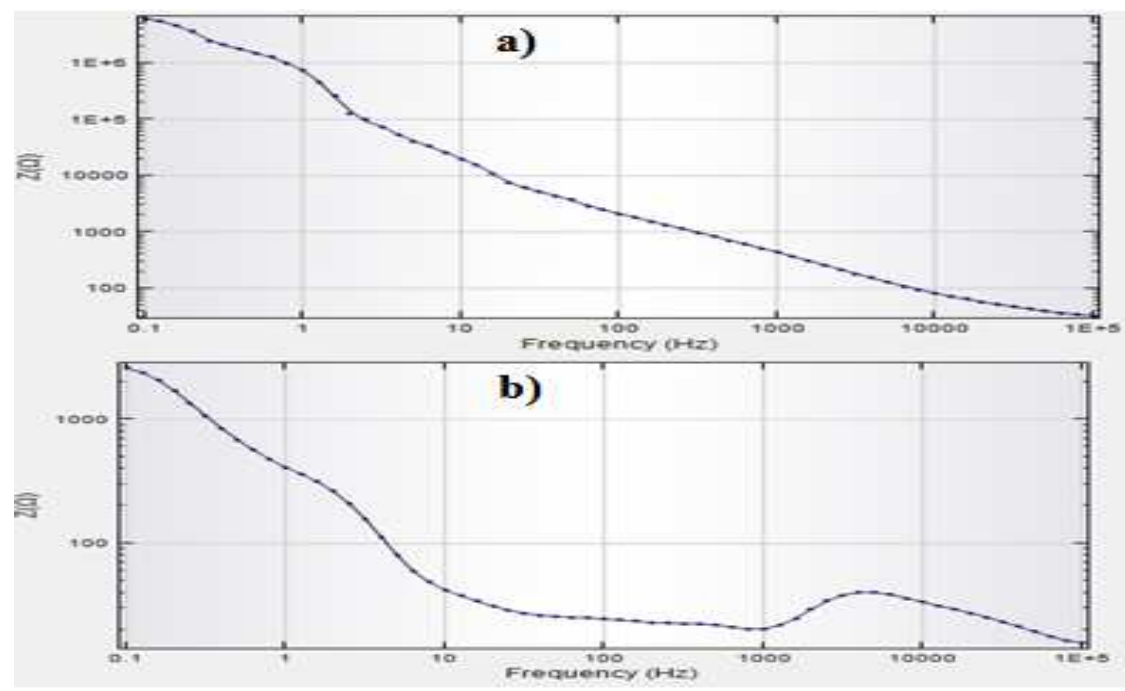

Figure 9. Bode modulus of (a) RGO/ITO and (b) RGO/RGO/ITO 

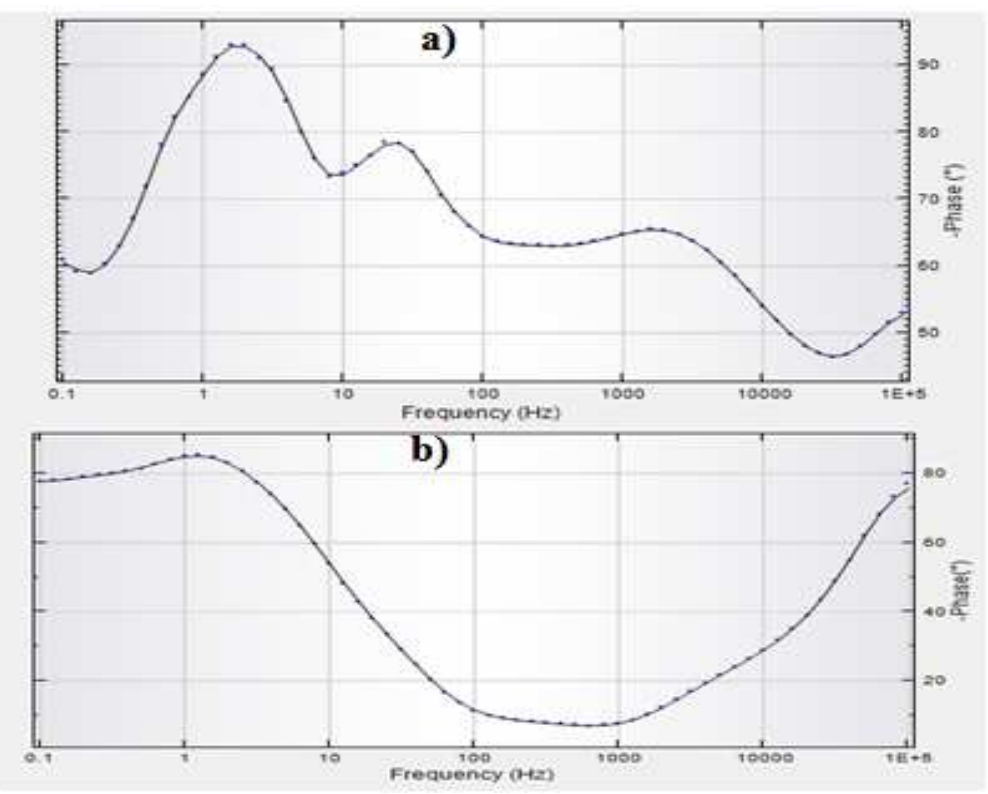

Figure 10. Bode phase for (a) RGO/ITO and (b) RGO/RGO/ITO using SG smoothing

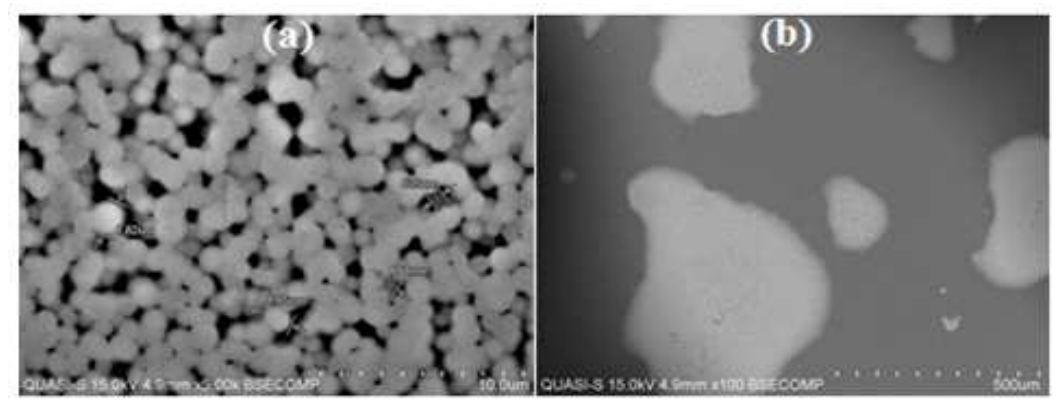

Figure 11. RGO/ITO (a) with magnification of 100 of electrochemical deposition electrode and (b) at $\times 5 \mathrm{k}$

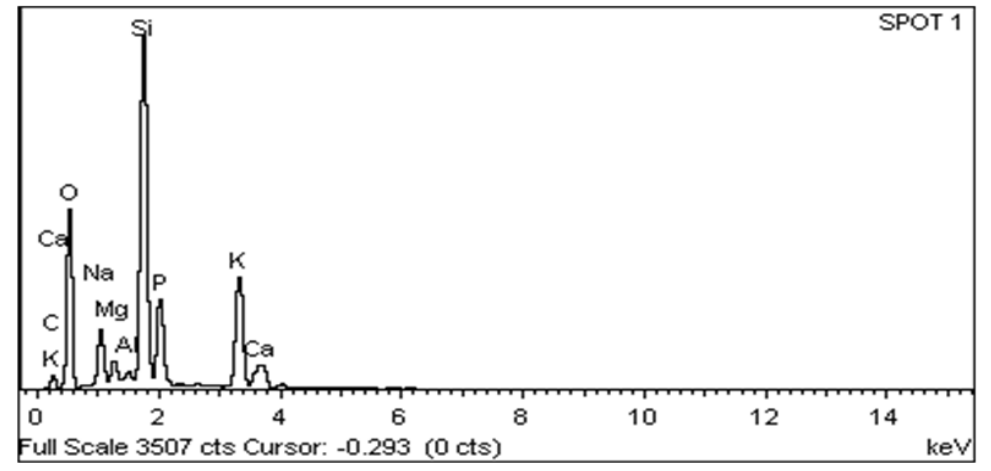

Figure 12. EDS elemental composition with applied $(\mathrm{kV})$ against the amount content

\section{Structural Analysis of Electrochemical Deposited} RGO Surface

Determining the effect of loads on the structure and its components using AFM in addition to proving successful electro-deposition of RGO on ITO glass substrate to examine nano-particles size, distribution and surface roughness; conducted on the samples in contact-mode for the topography of reduced graphene coated ITO glass substrates and the morphological changes were seen through the deposition of each layer. Table 2 shows the gradual increase in the surface roughness from ITO to the layers of RGO deposited. The magnification was set to an area of $(5 \times 5 \mu \mathrm{m})$ using contact mode. 
Table 1. AFM images of (a) ITO, (b) RGO/ITO, (c) RGO/RGO/ITO, (d)ITO/RGO/RGO at high scan rate of $1 \mathrm{Vs}^{-1}$ surface topology in (i) 2-D and (ii) 3-D

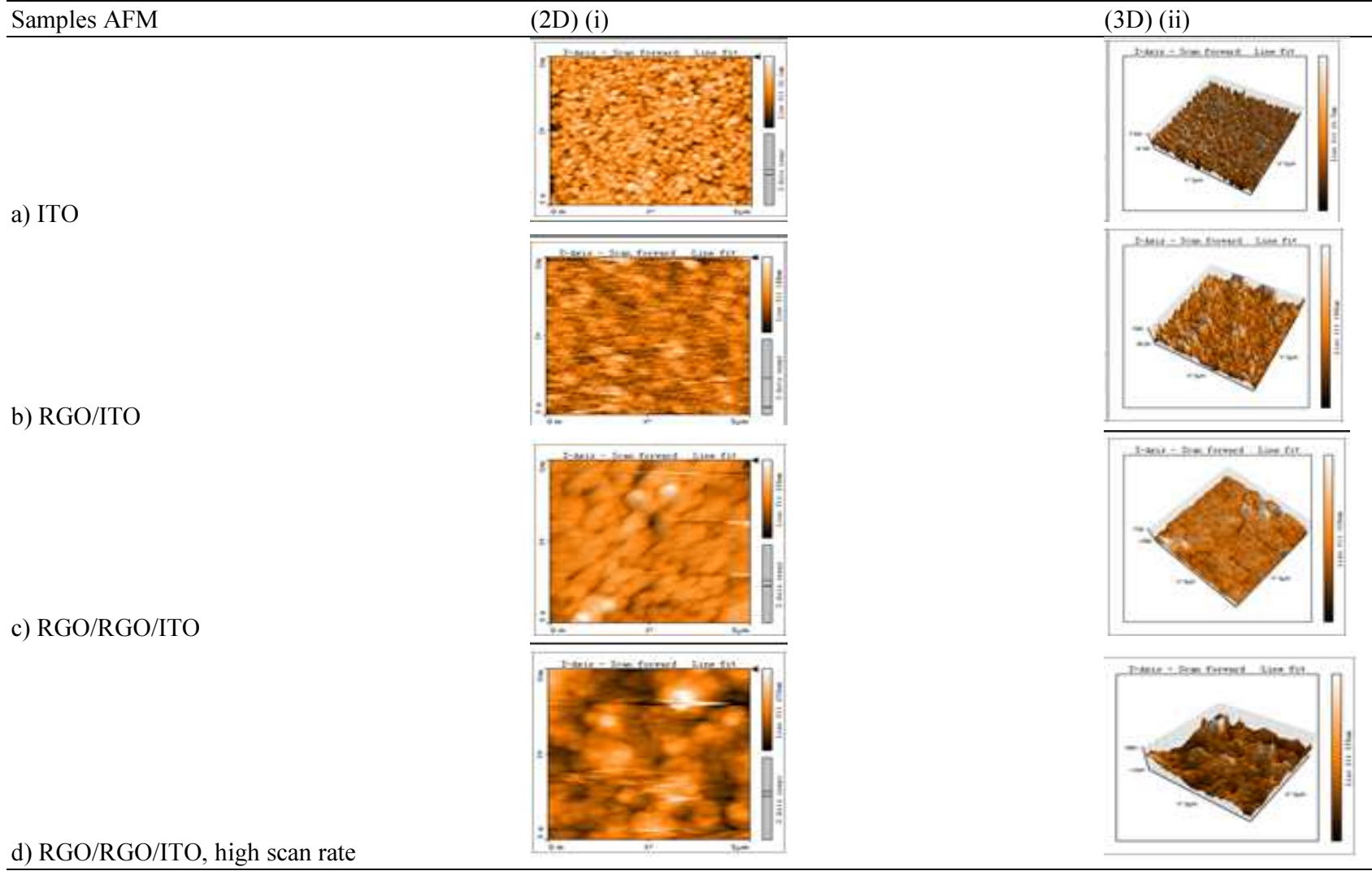

Table 2. Surface roughness of different samples from AFM analysis

\begin{tabular}{ll}
\hline Sample & $\mathrm{R}_{\mathrm{q}}$ (Surface roughness) $(\mathrm{nm})$ \\
\hline ITO & 2.773 \\
RGO/ITO & 19.116 \\
RGO/RGO/ITO & 34.93 \\
RGO/RGO/ITO high scan rate & 40.776 \\
\hline
\end{tabular}

Table 3. EDS records the samples elemental composition in weight

\begin{tabular}{lcc}
\hline Element & Weight\% & Atomic\% \\
\hline C K & 6.01 & 10.43 \\
O K & 41.20 & 53.56 \\
Na K & 4.22 & 3.82 \\
Mg K & 1.43 & 1.23 \\
Al K & 0.37 & 0.29 \\
Si K & 22.01 & 16.33 \\
P K & 7.88 & 5.30 \\
K K & 13.90 & 7.41 \\
Ca K & 2.98 & 1.55 \\
Totals & 100.00 & \\
\hline
\end{tabular}

Firstly, commercial ITO glass surface topography was seen as in Figure 11 (a)(i) $2 \mathrm{D}$ and (ii) $3 \mathrm{D}$ as for 11 (b) of RGO/ITO and (c) RGO/RGO/ITO at a slow scan rate of $1 \mathrm{~m} / \mathrm{Vs}^{-1}$ in comparison to a high scan rate of 1 $\mathrm{Vs}^{-1}$ respectively.

Table 1 records similar topology as for ITO circular particles that were slightly changed as a result of deposition wherein lighter regions were dedicated to higher surfaces and thus higher roughness while darker regioned owed to lower reagions. Never the less some white lines where due to noise and dust particles (Jampani et al., 2010; Miller, 2001).

Increasing the scan rate showed expected increased surface roughness as shown in Table 1 to $40.776 \mathrm{~nm}$ topology due to the graphene sheets agglomeration and increased noise signals. This agglomeration increased the surface roughness at the cost of reduced surface area.

\section{FESEM Images}

The sample was viewed under FESEM after electrodeposition of RGO in a 3-D visual in Figure 11 with (a) magnification of 100 and (b) magnification of $\times 5000$ of the surface presenting qualitative analysis with an average grain particles size range from $1.65 \mu \mathrm{m}$ to $635 \mathrm{~nm}$ which also proves the successful electrodeposition of Graphene over ITO.

The results showed that the ITO circular particles were cover with paper-like nanosheets, yet also non homogenous dispersion can be justified by agglomeration of graphene sheets due to high aspect ratio that can farther be reduced with surfactants for lowering the surface tension and increasing mono-dispersity of the size distribution. Finally, the samples EDS were also 
examined to determine the final samples elemental composition and the amount of impurities present in Figure 12. Also to presents of other chemicals such mainly oxygen from the atmosphere that binds to the surface's dangling bonds in absences of a vacuum to store the samples, moreover some impurities exist that might come from the synthesis method of Graphene oxide solution, such as potassium, phosphate, calcium and silicon and are shown in Table 3 along with their weight and atomic percentage.

\section{Conclusion}

ITO glass electrode substrates that were modified with graphene particles for different samples to form a uniform homogenous monolayer via a simple and costeffective, eco-friendly approach. Secondly the electrochemical properties of the modified electrodes were inspected separately in a three electrode system immersed in $0.1 \mathrm{M}$ phosphate buffer and $0.1 \mathrm{M} \mathrm{KCL}$ electrolyte at a $\mathrm{pH}$ of 7.2 for $\mathrm{CV}$, EIS and CDC properties where in the results showed success in high conductivity and ion transportation in the structural pores. In addition to UV-Vis characterization that showed decreased transmittance under visible light due to additional layers on substrates or increased concentration on GO and for monitoring the reduction process. Lastly but not least, the structural and morphological inspection with SEM and AFM to prove the success of the deposition process that also showed good structural and superior performance that was suggested as being a promising material in application of super capacitors and energy storage devices.

\section{Acknowledgement}

The authors would like to acknowledge the support from the Faculty of Engineering, Multimedia University, 63100 Cyberjaya, Malaysia for supporting the project activities.

\section{Funding Information}

This project is funded by Ministry of Higher Education (MOHE) through the Fundamental Research Grant (FRGS/MMUE-140051).

\section{Author's Contributions}

Mazin Ahmed Ali Moghazi: Conducted the experiments and drafted the article.

Sondos Al Shareef: Co-researcher on conducting the research experiments.

H.Y. Wong: Reviewed the article and co supervised the project.

Mukter Zaman: Project leader and corresponding author of the article.

\section{Ethics}

Authors hereby confirming that there are no conflict of interest with this manuscript.

\section{References}

An, S.J., Y. Zhu, S.H. Lee, M.D. Stoller and T. Emilsson et al., 2010. Thin film fabrication and simultaneous anodic reduction of deposited graphene oxide platelets by electrophoretic deposition. J. Phys. Chem. Lett., 1: 1259-1263. DOI: 10.1021/jz100080c

Beitollahi, H., S. Tajik, M.H. Asadi and P. Biparva, 2014. Application of a modified graphene nanosheet paste electrode for voltammetric determination of methyldopa in urine and pharmaceutical formulation. J. Analyt. Sci. Technol., 5: 29-29. DOI: $10.1186 / \mathrm{s} 40543-014-0029-y$

Chong, S.W., C.W. Lain and S.B.A. Hamid, 2015. Green preparation of reduced graphene oxide using a natural reducing agent. Ceram. Int., 41: 9505-9513. DOI: $10.1016 /$ j.ceramint.2015.04.008

Devadas, B., M. Rajkumar, S.M. Chen and R. Saraswathi, 2012. Int. J. Electrochem. Sci., 7: 3339-3349.

Han, Z.J., D.H. Seo, S. Yick, J.H. Chen and K.K. Ostrikov, 2014. $\mathrm{MnO}_{\mathrm{x}} /$ carbon nanotube/reduced graphene oxide nanohybrids as high-performance supercapacitor electrodes. NPG Asia Mater., 6: e140-e140. DOI: 10.1038/am.2014.100

Hibino, H., 2017. Graphene research at NTT. NTT Technical Review.

Jampani, P., A. Manivannan and P.N. Kumta, 2010. Advancing the supercapacitor materials and technology frontier for improving power quality. Electrochem. Society Interface, 19: 57-62. DOI: 10.1149/2.F07103 if

Lekakou, C., C. Lei, F. Markoulidis and A. Sorniotti, 2012. Nanomaterials and nanocomposites for high energy/high power supercapacitors. Proceedings of the 12th IEEE International Conference on Nanotechnology, Aug. 20-23, IEEE Xplore Press, Birmingham, pp: 1-7. DOI: $10.1109 /$ NANO.2012.6322011

Li, J. and M. Östling, 2013. Prevention of graphene restacking for performance boost of supercapacitors - a review. Crystals, 3: 163-190. DOI: $10.3390 /$ cryst3010163

Lia, X. and B. Weia, 2013. Supercapacitors based on nanostructured carbon. Nano Energy, 2: 159-173. DOI: 10.1016/j.nanoen.2012.09.008

Miller, J.R., 2001. Highly-reversible electrical energy storage: Technologies, applications and societal impact JME. Inc. Phys. Rev. Lett., 86: 3364-3364.

Shao, Y. and J. Wang, 2010. Facile and controllable electrochemical reduction of graphene oxide and its applications. J. Mater. Chem., 20: 743-748. DOI: 10.1039/B917975E 
Wang, K., L. Li and X. Wu, 2013. Synthesis of graphene and electrochemical performance. Int. J. Electrochem. Sci., 8: 6763-6766.

Yu, G., X. Xie, L. Pan, Z. Bao and Y. Cui, 2013. Hybrid nanostructured materials for high-performance electrochemical capacitors. Nano Energy, 2: 213-234. DOI: 10.1016/j.nanoen.2012.10.006

Zang, J. and X. Li, 2011. In situ synthesis of ultrafine $\beta$ $\mathrm{MnO}_{2}$ /polypyrrole nanorod composites for highperformance supercapacitors. J. Mater. Chem., 21: 10965-10969. DOI: 10.1039/C1JM11491C
Zhang, X.D. Zhang, Y. Chen, X.Z. Sun and Y.W. Ma, 2012. Electrochemical reduction of graphene oxide films: Preparation, characterization and their electrochemical properties. Chinese Sci. Bull., 57: 3045-3050. DOI: $10.1007 / \mathrm{s} 11434-012-5256-2$ 